\title{
The incidence, intensity and host morbidity of human parasitic protozoan infections in gastrointestinal disorder outpatients in Buea Sub Division, Cameroon
}

\author{
Judith V. Mbuh, Helen N. Ntonifor, James T. Ojong \\ Department of Plant and Animal Sciences, Faculty of Science, University of Buea, P.O. Box 63 Buea, Cameroon
}

\begin{abstract}
Background: Numerous protozoans inhibit the gastrointestinal tract of humans with the majority being either non-pathogenic commensals or of a type that may result in mild disease. However, some of these organisms can cause severe diseases under certain circumstances while others may become highly virulent and invasive causing potentially lethal systemic disease. This study investigated the prevalence, intensity and host morbidity of human intestinal protozoan infections in individuals living in the Buea Sub-Division, Cameroon.

Methodology: Random sampling was used to collect stool samples from 356 patients in a cross-sectional study. All samples were examined by formol-ether concentration and direct smear techniques. Data collected was analyzed and differences in proportions were determined using the Chi square $\left(\chi^{2}\right)$ test, Fisher's exact test, or analysis of variance where appropriate.

Results: It was found that $28.1 \%(100 / 356)$ of the sampled population were infected with protozoans. Females showed a higher infection rate $(29.7 \%$; 56/182) than males $(26.4 \%$; 46/174) and there was a significantly $(\mathrm{P}<0.001)$ higher prevalence in rural areas $(38.7 \%$; 55/142) than in urban areas $(21.0 \% ; 45 / 214)$. The 6 to 12 years age group had a significantly $(\mathrm{P}<0.05)$ higher infection rate $(42.9 \%$; 30/70). The total prevalence of intestinal protozoans was as follows: E. histolytica $(24.4 \%)$, E. coli $(11.2 \%)$ and G. lamblia $(0.6 \%)$. The most prevalent morbidity effects associated with intestinal protozoan infections were abdominal pains, dysentery and body weakness.

Conclusions: Since human intestinal parasitic infections are high in the study area, mass treatment of people with intestinal protozoans is strongly recommended, especially in the rural areas where the prevalence was very high.
\end{abstract}

Key words: Intestinal protozoans, prevalence, intensity, morbidity, Cameroon

J Infect Dev Ctries 2010; 4(1):038-043.

(Received 24 May 2009 - Accepted 15 October 2009)

Copyright $\odot 2010$ Mbuh et al. This is an open-access article distributed under the Creative Commons Attribution License, which permits unrestricted use, distribution, and reproduction in any medium, provided the original work is properly cited.

\section{Introduction}

Intestinal parasitic infections are among the most common infections in the world and are responsible for considerable morbidity and mortality [1]. The epidemiology of intestinal parasitic infections shows that these parasites are found in every age group and in both sexes. However, the incidence is high in some areas and in some age groups. Human intestinal parasitic infections have a worldwide distribution, with the greatest incidence and intensity occurring in developing countries [2]. Invasive amoebiasis is prevalent in certain areas of the world including West and South-east Africa, China, and Mexico [3]. The high occurrence of these parasites is often related to poverty, poor living conditions and hygiene, and inadequate sanitation and water supply [4]. In Turkey it was noted that the prevalence of pathogenic parasites was high among people who had no toilets in their houses [5]. In Pakdasht, Tehran Province, the highest infection rate $(41.5 \%)$ was related to protozoan parasites [6].

Entamoeba histolytica has been recovered worldwide and is more prevalent in the tropics and sub-tropics than in colder climates. However, in poor sanitary conditions in temperate and colder climates, infection rates have been found to equal that seen in the tropics [7]. In a related study in Ardabil Iran, a total of 10 species were identified with Giardia lamblia (14\%), Blastocystis hominis (10\%) and Entamoeba coli $(4.1 \%)$ being the most common parasites [8].

G. lamblia also has a worldwide distribution with an incidence rate of between $11 \%$ and $30 \%$. In the United States of America, it is now considered to be the most common intestinal parasite of man and the leading cause of diarrhoea due to protozoan infections in humans [9]. It is also the most frequently reported intestinal parasite in Peru [10]. 
Intestinal infections in general affect more than two-thirds of the human population and mostly children [7,11]. The intensity of infection is a major determinant of morbidity and approximately reflected in the number of characteristic cysts passed out in faeces [1]. There are four clinical forms of invasive intestinal amoebiasis, all of which are generally acute dysentery or bloody diarrhoea, fulminating colitis, amoebic appendicitis and amoeboma of the colon [12].

The main conditions and factors which are known to favour the transmission of human intestinal protozoan parasites exist in Buea Sub Division, Cameroon, and limited information has been documented in this area on the epidemiology of these parasites. This study was therefore conducted to provide data on the incidence, intensity and host morbidity of human parasitic protozoan infections in the Buea Health District.

\section{Materials and methods}

Study site

The study was performed at the Regional Hospital Annex, Buea. This is the main hospital which serves Buea and its environs in the south-west region of Cameroon. This area is found on the slope of Mount Cameroon. The study was divided into urban and rural zones as follows: urban areas included Buea Town, Sandpit, Bokwango, Great Soppo, Long Street, Bonduma, Molyko and Clerks quarters, while Muea, Sasse, Small Soppo, Mile 17, Bolifamba, Bova, Bomaka and Bonakanda were considered as rural regions.

\section{Study design and subject}

A cross-sectional study design was used. Ethical clearance for this study was obtained from the Regional Delegation of Public Health, Buea. Patients who presented at the outpatient department of the hospital between November 2006 and July 2007 were screened for recruitment criteria. Three hundred and fifty-six patients of both sexes aged 1 to 90 years with stomach disorders who accepted to participate in this study and gave their consent were enrolled. Each subject was given a screw-capped plastic bottle for collection of a faecal sample. Each bottle was properly labeled with the patient's identification code. The age, sex and locality of each participant were equally recorded.

\section{Formol- ether concentration technique for diagnosing intestinal parasites}

A small stool sample (0.2 grams) was placed in a mortar and mixed with $7 \mathrm{ml}$ of $10 \%$ formol saline. A sieve was used to filter the sample into a centrifuge tube and $3 \mathrm{ml}$ of diethyl ether added and shaken vigorously. The mixture was then centrifuged at 1,500 rpm for 2 minutes. A drop of Lugol iodine was then added to the deposit and observed under the microscope. Within 45 minutes of preparation the faecal smears were examined using the $\mathrm{x} 10$ and $\mathrm{x} 40$ objectives of the light microscope for identification and enumeration of protozoan cysts [13]. Infection intensities were expressed as cysts per gram (mean count $/ \times 40$ field). The different protozoan species were identified using standard identification tables [14].

\section{Determination of morbidity}

Morbidity effects due to the protozoan parasites were determined by physical examination of stool samples provided by each subject for the following: watery or bloody diarrhoea, dysentery, mucous in stool and the colour of stool sample.

\section{Statistical analysis}

The data were keyed in Excel (Microsoft Corporation, Redmond USA 2003) and exported to SPSS version 9.0 for further analysis. Descriptive statistics were presented as counts, percentages, geometric means and standard deviations. Differences in proportions were determined using the Chi-squared test or Fisher's exact test and differences in group means using Analysis of Variance (ANOVA). The level of significance was set at $\mathrm{P}<$ 0.05 .

\section{Results}

\section{Incidence and intensity of intestinal protozoans}

Out of a total of 356 outpatients examined, 100 $(28.1 \%)$ were infected with intestinal protozoan. Although females showed a slightly higher infection rate $(29.4 \%$; 54/182) than males $(26.4 \%$; 46/174) the difference was not significant $(\mathrm{P}>0.05)$. With respect to locality, the infection rate was higher in rural areas $(38.7 \%$; 55/142) compared to patients from urban areas $(21.0 \% ; 45 / 214)$ and this difference was highly significant $(\mathrm{P}<0.001)$. The incidence of intestinal protozoan was highest $(42.9 \% ; 30 / 70)$ in the age group 6 to 12 years followed by 13 to 19 years $(29.2 \% ; 14 / 48)$ and the least incidence was seen in the age group 20 to 30 years $(21.6 \% ; 21 / 97)$. The 
Table 1. The incidence of mixed intestinal protozoan infection in the study population.

\begin{tabular}{|c|c|c|c|c|}
\hline \multirow[b]{2}{*}{ Variable } & \multicolumn{4}{|c|}{ Mixed Protozoan infections } \\
\hline & $\begin{array}{c}\text { No. infected } \\
\text { with protozoan }\end{array}$ & $\begin{array}{l}\text { No. with } \\
\text { mixed infection }\end{array}$ & $\begin{array}{c}\text { Prevalence } \\
(\%)\end{array}$ & P value \\
\hline Sex: & & & & \\
\hline Male & 46 & 16 & 34.8 & 0.001 \\
\hline Female & 56 & 15 & 26.8 & \\
\hline Locality: & & & & \\
\hline Rural & 55 & 20 & 36.4 & 0.433 \\
\hline Urban & 45 & 11 & 24.4 & \\
\hline $\begin{array}{l}\text { Age group } \\
\text { (years): }\end{array}$ & & & & \\
\hline $1-5$ & 08 & 02 & 25.0 & \\
\hline $6-12$ & 30 & 10 & 33.3 & 0.123 \\
\hline $13-19$ & 14 & 06 & 42.9 & \\
\hline $20-30$ & 21 & 04 & 19.0 & \\
\hline$>30$ & 27 & 09 & 33.3 & \\
\hline
\end{tabular}

difference in incidence between the age groups was also significant $\mathrm{P}<0.05$ (Figure 1).

The overall geometric mean intensity of infection was 392 cysts per gram (cpg) of faeces. Although females had a higher geometric mean parasite density (424 cpg) than males (358cpg), the difference was not significant $(\mathrm{P}>0.05)$. The geometric mean parasite density was higher in rural areas (404cpg) than in urban regions (376cpg). The geometric mean parasite density followed the same trend as incidence, with age group 6 to 12 years having the highest geometric mean parasite density of $435 \mathrm{cpg}$ of faeces; the least was seen in age group 20 to 30 years with a geometric mean intensity of $336 \mathrm{cpg}$ of faeces (Figure 1). The difference in geometric mean parasite density in the different age groups was significant $(\mathrm{P}$ $<0.05)$.

\section{Incidence and intensity of the different species of} intestinal protozoans

The incidence of the different species of intestinal protozoan is shown in Figure 2. Three species of protozoans were encountered in the study viz: E. histolytica with infection rate of $24.4 \%$, followed by $E$. coli $(11.2 \%)$ and the least was $G$. lamblia with an infection rate of $0.6 \%$. In the different age groups, the incidence of $E$. histolytica followed by $E$. coli were higher in patients between 6 and 19 years of age and least in subjects between 20 to 30 years old. The geometric mean parasite densities were $345 \mathrm{cpg}, 226 \mathrm{cpg}$ and $32 \mathrm{cpg}$ of faeces for E. histolytica, E. coli and G. lamblia respectively (Figure 2). Figure 3 shows that the infection with $E$. histolytica and $E$. coli was higher in females than in males. More people were infected with E. histolytica in the rural areas $(35.9 \% ; 51 / 142)$ than in urban areas $(16.8 \% ; 36 / 214)$ and this difference was found to be highly significant $(\mathrm{P}<0.001)$. The same trend was seen in $E$. coli with patients from rural areas having a higher incidence $(16.9 \% ; 24 / 142)$ when compared to those from urban areas $(7.5 \% ; 16 / 214)$. The difference was also significant $(\mathrm{P}<0.01)$. Only two cases of G. lamblia were recorded in this study and they were only found in stool samples provided by patients from urban areas.

The intensity of protozoan cysts was found to be higher in females than in males (Figure 4). Similarly, patients from rural areas had higher parasite load (354cpg) than those from urban areas (332cpg) for $E$. histolytica while that for $E$. coli was higher in urban areas $(289 \mathrm{cpg})$ than in rural areas (192cpg) (Figure 4). In both species, these differences were not 
Figure 1. The relationship between prevalence and intensity of protozoan infection in the study population.

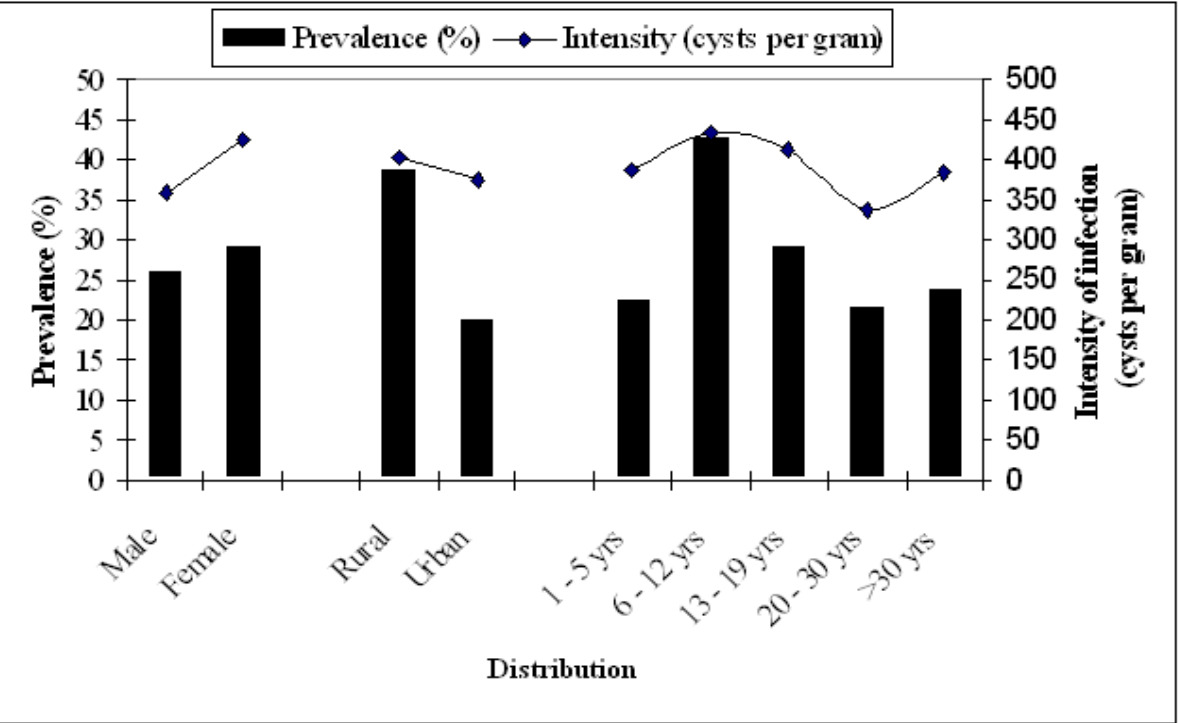

Figure 3. Prevalence of the different protozoan species in relation to sex, locality and age group.



Figure 2. The prevalence and intensity of different protozoan species in the study population.

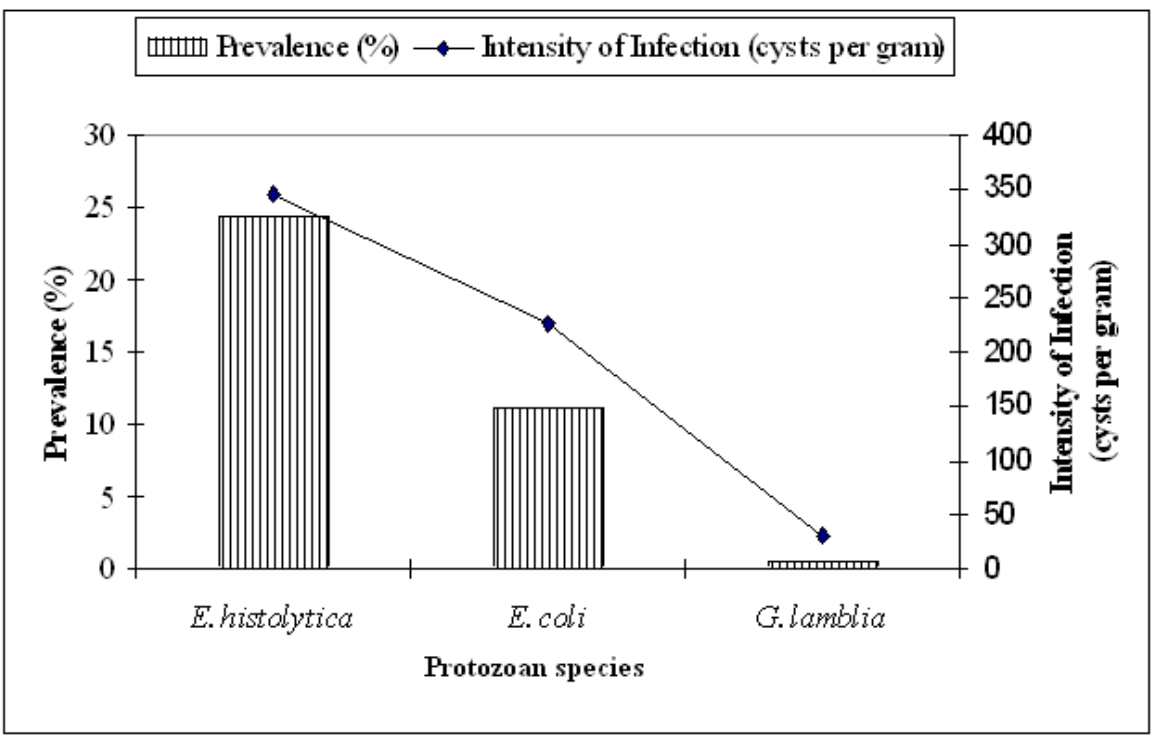

Figure 4. The intensity of the different protozoan species in relation to sex, locality and age group

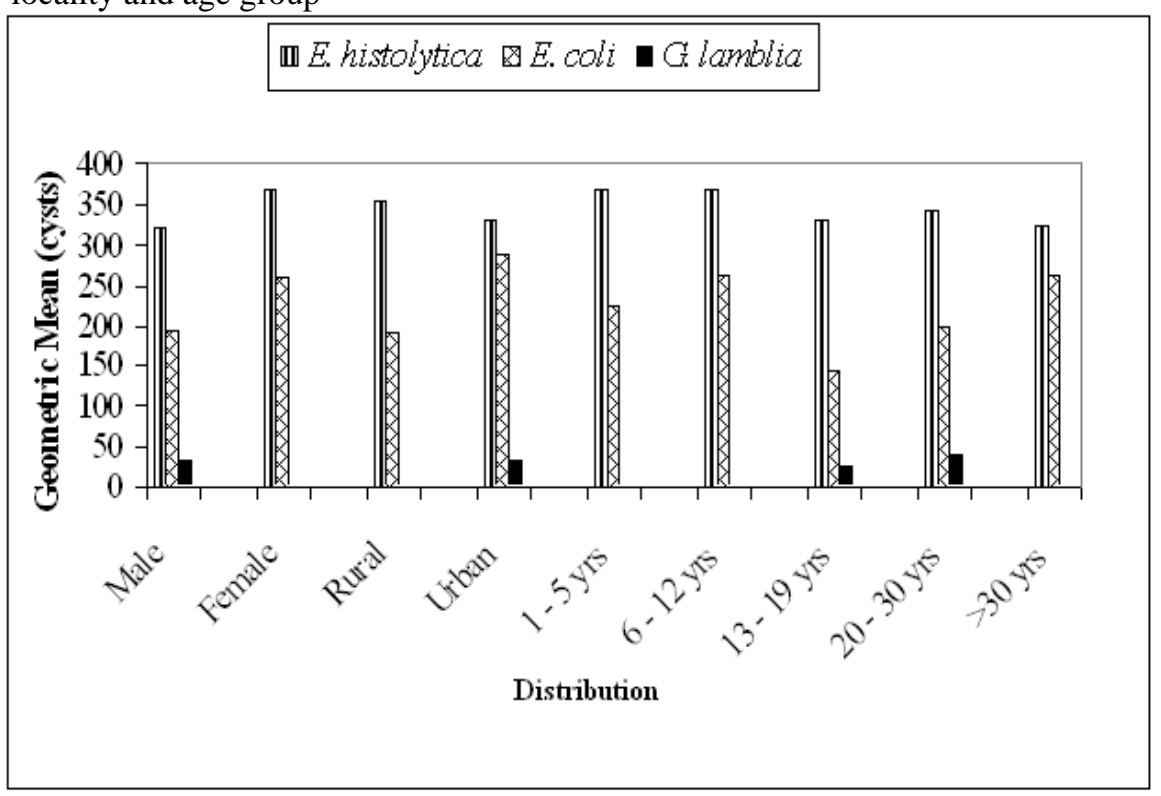


significant. Figure 4 also shows that the highest intensity of infection with E. histolytica was seen in age group 6 to 12 years $(367 \mathrm{cpg})$ and the least in the group younger than 30 years $(324 \mathrm{cpg})$. The difference in the intensity of infection in the different age groups was very significant $(\mathrm{P}<0.001)$.

\section{Relationship between the incidence and intensity of infection with intestinal protozoans}

There was a positive relationship between the incidence and intensity of infection with human intestinal protozoans in this study. Females had a higher incidence and intensity than males. The same trend was observed in patients from rural areas having a higher incidence and intensity of infection than those from urban areas. In the different age groups, there was a highly positive correlation (+1.0) between the incidence and intensity of infection with intestinal protozoans.

Out of 100 samples infected with protozoans, mixed infections were seen in 31 cases $(31.0 \%)$. The incidence of mixed infections was higher in males $(34.8 \%$; 16/46) than ini females $(27.8 \% ; 15 / 56)$. Equally, the incidence was higher in rural areas $(36.4 \% ; 20 / 55)$ than in urban areas $(24.4 \% ; 11 / 45)$. The difference was highly significant $(\mathrm{P}<0.001)$ (Table 1). In the different age groups, the highest incidence with respect to ages was seen between 13 and 19 years $6(42.9 \%)$, while the least was in age group 20 to 30 years $4(19.0 \%)$.

\section{Morbidity effects due to intestinal protozoans}

All the 100 cases infected with protozoans presented with one or more clinical signs and symptoms. The highest occurrence $(83.3 \%$; 45/54) of clinical symptoms was recorded in females as compared to males $(80.4 \% ; 37 / 46)$. The morbidity effects were higher in patients from urban areas $(88.9 \% ; 37 / 45)$ than in patients from rural areas $(81.8 \%$; 45/55). A high incidence was observed in study subjects above 30 years of age $(85.2 \% ; 23 / 27)$ while the lowest incidence $(62.5 \% ; 5 / 8)$ was observed in patients less than 6 years of age. Dysentery and abdominal pains were the highest morbidity effects recorded in the study population.

\section{Discussion}

Results of this survey in Buea and its environs indicated that the transmission of intestinal protozoan is not only active, but it is on the rise. E. histolytica, E. coli and G. lamblia were the intestinal protozoan observed in the study area. In the sex-related incidence and intensity of infections, females recorded a higher incidence and intensity of infections than males. This difference could be due to the fact that females are more engaged in farming activities and these farms are often contaminated by promiscuous defaecation. The higher incidence rate of human intestinal protozoan in females than in males could also be attributed to the fact that women usually eat unwashed fruits and vegetables in the farms which may be contaminated with cysts of protozoan, thus facilitating their acquisition of the infections.

Patients from rural areas also showed a higher incidence rate than those from urban centres. This is often related to poverty and the poor living and hygienic conditions prevailing in the rural areas compared to urban areas. The study also revealed a significant positive correlation between incidence and intensity of infection among different age groups with peak values among the 6 to 12 years age group. Similar results were reported in Simbok, Cameroon [7]. With respect to the different species of protozoan identified, E. histolytica and E. coli showed a relatively higher incidence than $G$. lamblia. These results are similar to those reported in other regions of Cameroon [7,15], but they are not in line with those obtained by other authors who found that the most prevalent protozoan species was $G$. lamblia $[8,10]$. The high incidence of $E$. histolytica and $E$. coli in our study could be due to the existence of resistant cysts of both parasites species in our study area. G. lamblia was very scarce in the study area; the two cases reported could have been imported from another area. Single and mixed protozoan infections of $E$. histolytica and $E$. coli constituted $69.0 \%$ and $31.0 \%$ respectively. Earlier studies indicated that multiple and mixed parasite infections are often encountered in children from endemic areas in developing countries [16,17].

In the major protozoan infections, an estimate of intensity is of little value and the central parameter for evaluation is incidence. Incidence exhibits age and spatial heterogeneity, which may be speciesspecific, so there remains a need to ensure a consistent sample structure, although this is less critical than for the helminthes [18].

Eighty-two $(82.0 \%)$ of the 100 cases infected with one or more species of protozoan presented with at least one clinical symptom caused by protozoans. This was relatively very high, especially as the majority of our study subjects complained of stomach-related problems at recruitment. The 
morbidity effect was not affected by sex or locality as both males and females and patients from rural and urban areas had approximately the same occurrence of morbidity effects. The most prevalent morbidity effects seen in this study were abdominal pains, dysentery and body weakness. Abdominal pains may result following invasion of the stomach wall by $E$. histolytica.

Based on our findings, it is evident that the incidence of intestinal protozoan infections is relatively high in our study area, with very heavy parasite loads being concentrated in a few individuals. This therefore highlights the need to educate members of the community, especially those in rural areas, on the methods of preventing the transmission and spread of these human intestinal protozoan infections.

\section{References}

1 . Kongs A, Marks G, Verle P, Van Der, Stuyft P (2001) The Unreliability of the Kato-Katz Technique Limits its Usefulness for Evaluating S. mansoni Infections. Tropical Medicine and International Health 6: 163-169

2. Naish S, McCarthy J, Williams GM (2004) Prevalence, Intensity and Risk Factors for Soil-transmitted Helminth Infections in a South Indian Fishing Village. Acta Tropica 91: 177-187.

3. Mirelman D (1987) Amoeba-bacterial Relationship in Amoebiasis. Microbiol Rev 51: 272-284.

4. Feenstra S, Raheela H, Wim van der Hoek (2000) Prevalence of Intestinal Parasites in the Southern Punjab, Pakistan. Pakistan Journal of Health 5: 3234.

5. Aksoy U, Akisu C, Tuncay S, Delibas SB, Iceboz T, Over L, Oral A M (2005) An Outbreak of Intestinal Protozoa Associated with Drinking Water. Journal of Science and Medicine 73: 163.

6. Schabazi F, Nourjah N, Baniardalan M (2003) The study of prevalence of intestinal parasites in Pakdasht, University of Medical School. Annals of Tropical Medicine and Parasitology 84: 373.

7. Kouontchou S, Quakyi IA, Befidi RM (2002) Prevalence of Multiple Concomitant Intestinal Infections in Simbok, a Malaria Endemic Village in Cameroon. Journal of the Cameroon Academy of Sciences 2: 202-296.

8. Daryani A, Eltehad GS, Ziael H (2003) Prevalence of Intestinal Parasitic Infections among Primary School
Students in Ardabil. Journal of Ardabil University of Medical Sciences 5: 229-234.

9. Huang DB, White AC (2006) An Updated Review on Cryptosporidium and Giardia. Gastroenterol Clin. North America 35: 291-314.

10. Beltran M, Garaycochea M, Bellido N, Garcia J, Rios L, Bernui G, Gonzales R (2004) Prevalence of Amoebiasis by Entamoeba histolytica / E. dispar in Three Regions of Peru. National Institute of Health 8: 316.

11. Uneke CJ, Eze KO, Oyibo PG, Azu NC, AMLT EA (2007) Soil Transmitted Helminth Infections in School Children in South East Nigeria: The Public Health Implication. Internet Journal of Third World Medicine ISSN: 1539-4646.

12. Renmert W, Ray C (2000) Fulminant Amoebic Colitis in Ten Day Old Infection. Journal of Pediatric Infection Diseases 19: 111-112.

13. Christensen N O, Gotsche G, Frandsen F (1984) Parasitological Techniques for Use in

Laboratory Maintenance of Schistosomes and Used in the Studies of the Epidemiology of Human and Bovine Schistosomiasis. Danish Bilharziasis Laboratory Manual. 112 pp.

14. Anonymous (1997) Amoebiasis. Wkly Epidemiol Rep. 72: 97-100.

15. Rossignol JF, Ayoub A, Ayers MS (2001) Treatment of Diarrhoea Caused by Giardia intestinalis and Entamoeba histolytica or E. dispar: A Randomized, Double-Blind, Placebo - Controlled Study of Nitazoxanide. Journal of Infectious Diseases 184: 381 - 384.

16. Hohmann H, Panzer S, Phimpachan (2001) Relationship of Intestinal Parasites to the Environment and to Behavioural Factors in Children in the Bolikhamxay Province of Lao PDR. South East Asian Journal of Tropical Medicine and Public Health 32: 4.

17. Lopiso E, Yared M, Ayele A (2002) Prevalence of Hookworm Infection and Haemoglobin Status among Rural Elementary School Children in Southern Ethiopia. Ethiopian Journal of Health and Development 16: 113.

18. Bundy DAP, Hall A, Medley GF, Savioli L (2004) Evaluating Measures to Control Intestinal Parasite Infections. World Health Stat Q 45: 168.

\section{Corresponding Author}

Judith V. Mbuh

Department of Plant and Animal Sciences, Faculty of Science University of Buea

PO Box 63 Buea, Cameroon

Tel: +237 77523087 Fax: +2373332 2272

Email: judithmbuh@yahoo.com

Conflict of interest: No conflict of interest is declared. 